\title{
Erratum
}

\section{Calculation of the Surface Parallel Critical Field for Clean Superconducting Films}

\author{
GERHART LÜDERS
}

Z. Physik 209, 219 (1968)

The signs in Eq. (56) are incorrect. This equation should read

$$
\begin{aligned}
B= & -p \frac{45 \zeta(4)}{224 \zeta(3)} \eta\left[\left(\xi^{2} u^{2}\right)_{\xi_{l}}+\left(\xi^{2} u^{2}\right)_{\xi_{r}}\right] \\
& -\frac{31 \zeta(5)}{70 \zeta(3)} \eta^{2}\left[\left(\xi u^{2}\right)_{\xi_{l}}-\left(\xi u^{2}\right)_{\xi_{r}}\right]+\cdots
\end{aligned}
$$

As a consequence, Eq. (69) is changed into

$$
2 \frac{d \eta}{\eta}-\frac{A(\varepsilon=1)}{\tau}= \begin{cases}\frac{93 \zeta(5)}{70 \zeta(3)}\left(\frac{\xi_{0}(T)}{d}\right)^{2}+\cdots & (p=0), \\ p \frac{135 \zeta(4)}{224 \zeta(3)} \frac{\xi_{0}(T)}{d}+\cdots & (p>0)\end{cases}
$$

where we have dropped the temperature dependent terms which, in a consistent approximation, should not have been written down. Finally, Eq. (12) is to be replaced by

$$
\frac{H_{C \mid !}(T)}{H_{C 2}(T)} \frac{d}{\sqrt{7 \zeta(3)} \xi_{\Delta}(T)}=1+ \begin{cases}\frac{93 \zeta(5)}{70 \zeta(3)}\left(\frac{\xi_{0}(T)}{d}\right)^{2}+\cdots & (p=0), \\ p \frac{135 \zeta(4)}{224 \zeta(3)} \frac{\xi_{0}(T)}{d}+\cdots & (p>0) .\end{cases}
$$

I wish to thank K.D. USADEL, who, by the results of his own work ${ }^{1}$, suggested another check of my previous results. H.J. Sommers' assistance in the calculations is gratefully acknowledged.

\footnotetext{
${ }^{1}$ UsADEL, K.D.: Z. Physik, to be published.
}

Prof. Dr. G. LÜDERS

Institut für Theoretische Physik der Universität

3400 Göttingen, Bunsenstraße 9 